\title{
HUMAN PANTOTHENIC ACID DEFICIENCY PRODUCED BY OMEGA-METHYL PANTOTHENIC ACID *
}

\author{
By ROBERT E. HODGES, WILLIAM B. BEAN, MARGARET A. OHLSON AND \\ ROBERTA BLEILER \\ (From the Metabolic Section of the Department of Internal Medicine, University Hospitals, \\ College of Medicine, State University of Ioz'a, Iozva City, Ioz'a)
}

(Submitted for publication March 3, 1959; accepted April 30, 1959)

Since the discovery that pantothenic acid comprised a major portion of coenzyme $\mathrm{A}$, this vitamin has been studied intensively by workers in biology and nutrition as well as in clinical medicine. It was found to be essential for the growth, health and reproduction of many species of fowls and mammals. Its importance in human nutrition remained long in dotibt, however, because of its presence in nearly all foods eaten by man. In 1954 we reported that pantothenic acid was essential in human nutrition. This study and subsequent ones demonstrated that young men fed a purified, partly synthetic diet deficient in pantothenic acid, became ill after about 12 weeks (1, $2)$. By adding omega-methyl pantothenic acid to the deficient diet, we were able to induce illness in about four weeks (3-5). The details of this illness and of the unpredictable results stemming from unforeseen peculiarities of the test program have been described (4-6). After correction of defects in the experimental plan, we established the characteristics of pantothenic acid deficiency in man. The symptoms included fatigue, headache, weakness, emotional lability, impaired motor coordination, paresthesias, muscle cramps and gastrointestinal disturbance such as nausea, vomiting and abdominal cramps. Some subjects had tachycardia, orthostatic hypotension and fluctuations in arterial blood pressure. The subjects developed an increase in the erythrocyte sedimentation rate, eosinopenia and an inability to respond to adrenocorticotropic hormone with further eosinopenia. They became increasingly sensitive to the hypoglycemic effect of insulin. Interpretation of these results was modified with each successive study. In some respects the findings

* This project was supported in part by grants from the National Institutes of Health, The American Cancer Society, The Nutrition Foundation and the National Vitamin Foundation. suggested the same adrenocortical insufficiency which occurs in animals deficient in pantothenic acid, yet many features were missing from the human counterpart. Whatever the mechanism, our subjects were ill as a result of pantothenic acid deficiency. Most recovered promptly when large doses of this vitamin were given.

\section{METHODS}

Four healthy young men were selected from prison volunteers. They ranged in age from 22 to 29 years, had been healthy all their lives, and were normal by physical examination (Table I). Their blood, urine, X-rays of the chest and electrocardiograms were normal. All were fed a general diet containing 3,200 calories and $120 \mathrm{Gm}$. of protein throughout the study. Calculations of the amount of pantothenic acid in this diet averaged $8.484 \mathrm{mg}$. daily (Table II). The regimen for each man differed from that of his fellows only in the amount of the anti-vitamin which he received. One man received none, another got $0.5 \mathrm{Gm}$. daily, a third $1 \mathrm{Gm}$. daily, and the fourth $2 \mathrm{Gm}$. daily. At the beginning of the seventh week of the test, the dose of the antagonist was doubled so that Subject No. 2 received $1 \mathrm{Gm}$.; No. 3, $2 \mathrm{Gm}$.; and No. 4, $4 \mathrm{Gm}$. daily. This schedule was maintained into the twelfth week when it was discontinued. Thereupon $4 \mathrm{Gm}$. of pantothenic acid was given to each man every day.

The men were under constant supervision. They were interviewed daily by R.E.H. or W.B.B. We made routine observations of their general condition, blood pressure, pulse and respiratory rates. They were weighed daily. Complete collections were made of urine and feces for nitrogen determination to compare with aliquots of the daily diet. Determinations were made each

TABLE I

Description of subjects

\begin{tabular}{ccccl}
\hline \hline Subject & Age & Height & Weight & \multicolumn{1}{c}{ Program } \\
\hline No. & yrs. & inches & lbs. & \\
1 & 22 & 70 & 144 & Control \\
2 & 29 & 71 & 154 & Antagonist, 0.5-1.0 Gm./day \\
3 & 24 & 68 & 161 & Antagonist, 1.0-2.0 Gm./day \\
4 & 23 & 72 & 164 & Antagonist, 2.0-4.0 Gm./day
\end{tabular}


TABLE II

Diet content of pantothenic acid*

\begin{tabular}{cc}
\hline \hline Date & Total pantothenic acid \\
\hline & $m g$. \\
$9-30-57$ & 8.615 \\
$10-13-57$ & 8.645 \\
$10-17-57$ & 7.939 \\
$10-25-57$ & 8.399 \\
$11-1-57$ & 8.272 \\
$11-11-57$ & 8.068 \\
$11-22-57$ & 8.484 \\
$12-2-57$ & 9.357 \\
Average & 8.484
\end{tabular}

* Figures taken from Zook, E. G., MacArthur, M. J., and Toepfer, E. W. Pantothenic acid in foods. U.S.D.A. Agriculture Handbook No. 97, 1956.

week of serum proteins by paper electrophoresis, sedimentation rate, blood counts and urine analysis. Special tests ${ }^{1}$ included a Thorn test (eosinopenic response to adrenocorticotropic hormone) (ACTH) and an insulin tolerance test ( 0.1 unit per $\mathrm{Kg}$. body weight subcutaneously) once each week. Every second week bromsulphalein tests (BSP) and serum bilirubin determinations were made to evaluate hepatic function. Electrocardiograms were taken every second week.

\section{RESULTS}

Clinical observation disclosed the following. The control subject, No. 1 , had virtually no

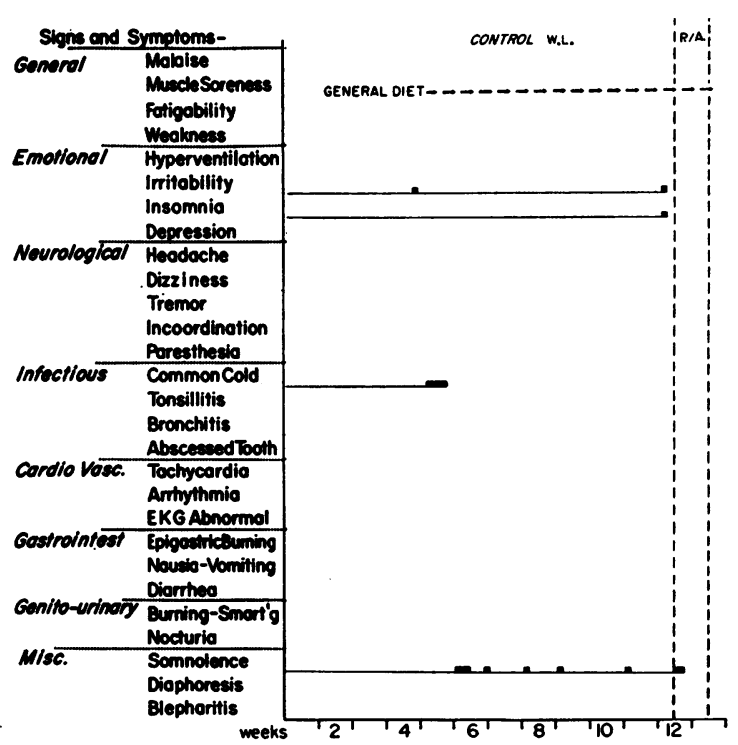

Fig. 1. Clinical Aspect of Pantothenic Acid DeFiciency-Control Subject No. 1

Subject remained well throughout the study.

${ }^{1}$ By the same methods used in previous studies (1-6).

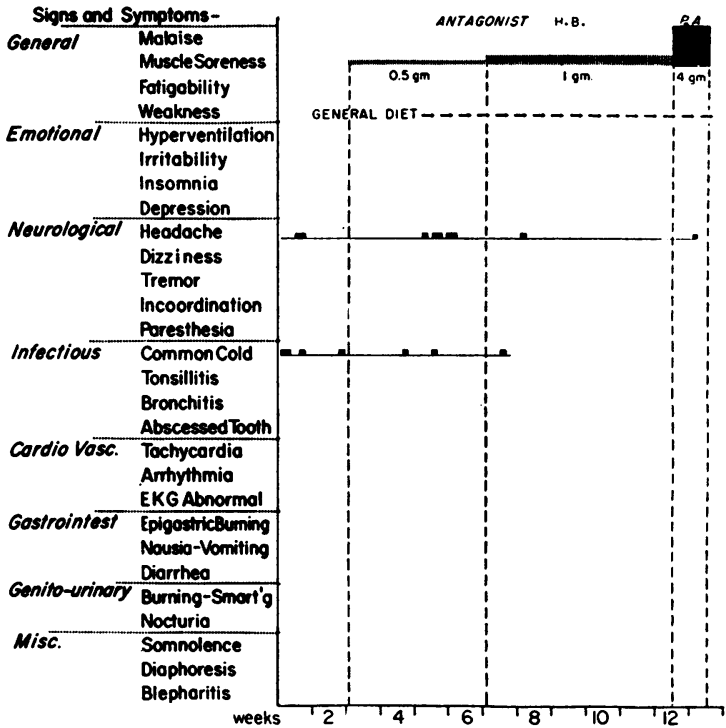

Fig. 2. Clinical Aspect of Pantothenic Acid DeFICIENCY-SUBJECT No. 2

Subject No. 2 was given the antagonist, $0.5 \mathrm{Gm}$. daily, from the third to the seventh week, at which time the dose was $1.0 \mathrm{Gm}$. daily until the twelfth week. He remained quite well.

symptoms throughout the entire study period (Figure 1). On occasions he was slightly irritable. Once he had a cold and for a few days he was drowsy, but his physical examination remained normal. Subject No. 2 who received the smallest dose of omega-methyl pantothenic acid complained on several occasions of a headache which was not severe (Figure 2). He had several colds but his physical state remained normal. Subject No. 3 was well during the first four and onehalf weeks of the experimental period (Figure 3 ). After this he had minor complaints including a mild headache, a cold and, on one occasion, slight nausea, but his physical examination remained normal. After the antagonist was increased to $2 \mathrm{Gm}$. a day he began to feel ill and was somnolent much of the time. In a few weeks he became irritable and depressed, desiring to be left alone. Later he developed a tremor of the outstretched hands. He had nausea with occasional episodes of vomiting. Administration of pantothenic acid in a dose of $4 \mathrm{Gm}$. daily was soon followed by alleviation of his symptoms. Subject No. 4, in spite of receiving the largest dose of the anti-vitamin, had no symptoms during the first phase of the study (Figure 4). But when the 


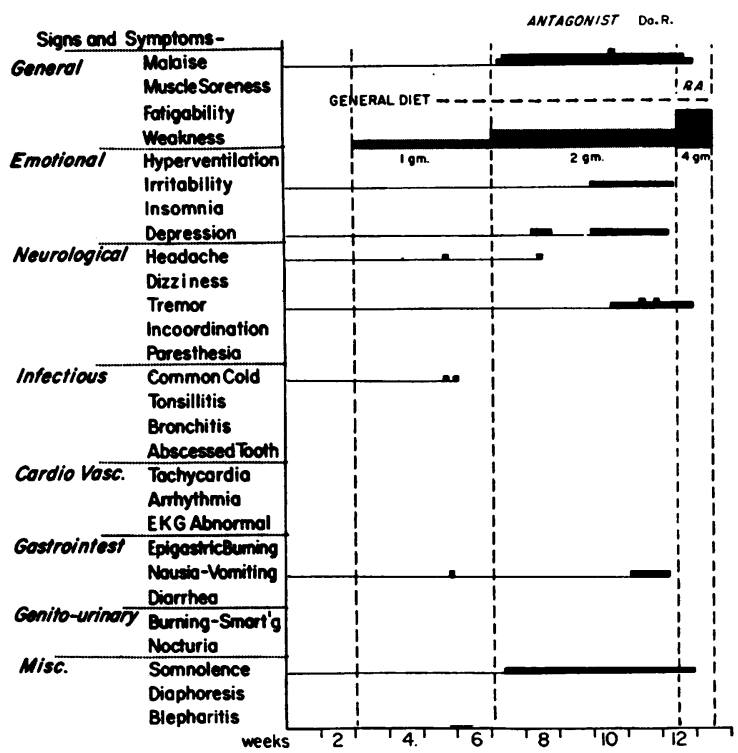

Fig. 3. Clinical Aspect of Pantothenic Acid DeFICIENCY-SubJeCt No. 3

Subject No. 3 was given $1 \mathrm{Gm}$. of the antagonist daily from the third to the seventh weeks, and $2 \mathrm{Gm}$. daily until the twelfth week. At this time $4 \mathrm{Gm}$. of pantothenic acid was given daily. He became ill during the seventh week and improved rapidly after pantothenic acid was given.

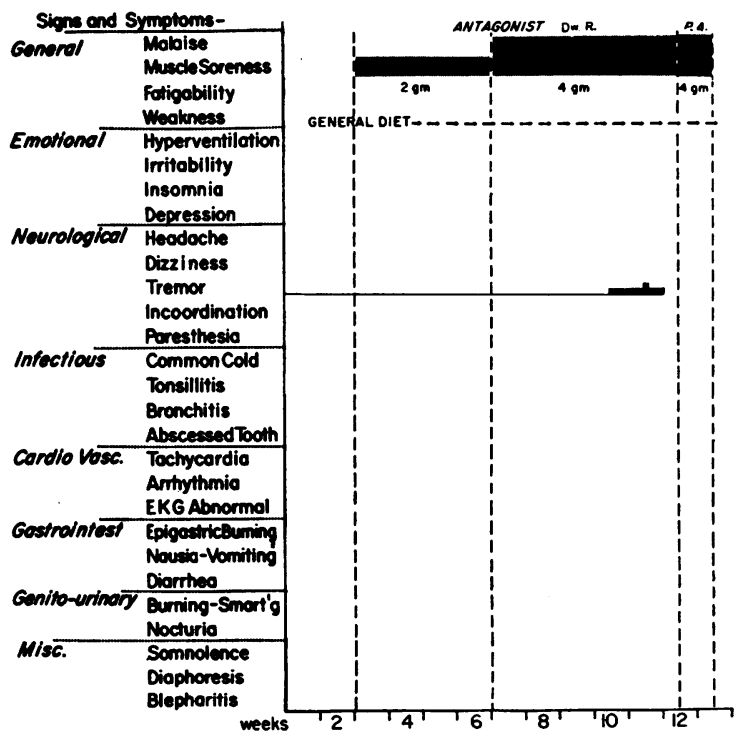

Fig. 4. Clinical Aspect of Pantothenic Acid DeFICIENCY-SUBJECT No. 4

Subject No. 4 was given $2 \mathrm{Gm}$. of the antagonist daily from the third to the seventh weeks and $4 \mathrm{Gm}$. daily until the twelfth week. He remained well except for a tremor. This subsided spontaneously before pantothenic acid was given, and did not return. dose was doubled to $4 \mathrm{Gm}$. daily he developed a tremor of his outstretched hands as the sole abnormality. This disappeared spontaneously a short time before the administration of the vitamin and it did not recur.

None of the subjects developed abnormalities in body weight, temperature, pulse, blood pressure or respiratory rate. The white blood counts, differential counts, hematocrits, urinalyses, electrocardiograms, chest X-rays and the hepatic function studies (BSP and serum bilirubin) remained normal. The erythrocyte sedimentation rate remained normal in Subject No. 1, increased slightly in Subjects No. 2 and 3 and did not change in Subject No. 4. The eosinopenic response to ACTH remained normal in Subjects one and two. In Subject 3 a noticeable decrease in the total eosinophil count developed after he received the antagonist. During the tenth week his eosinopenic response to ACTH became impaired, but recovered and remained normal after pantothenic acid was administered. The man given the largest dose of pantothenic acid antagonist had a sharp

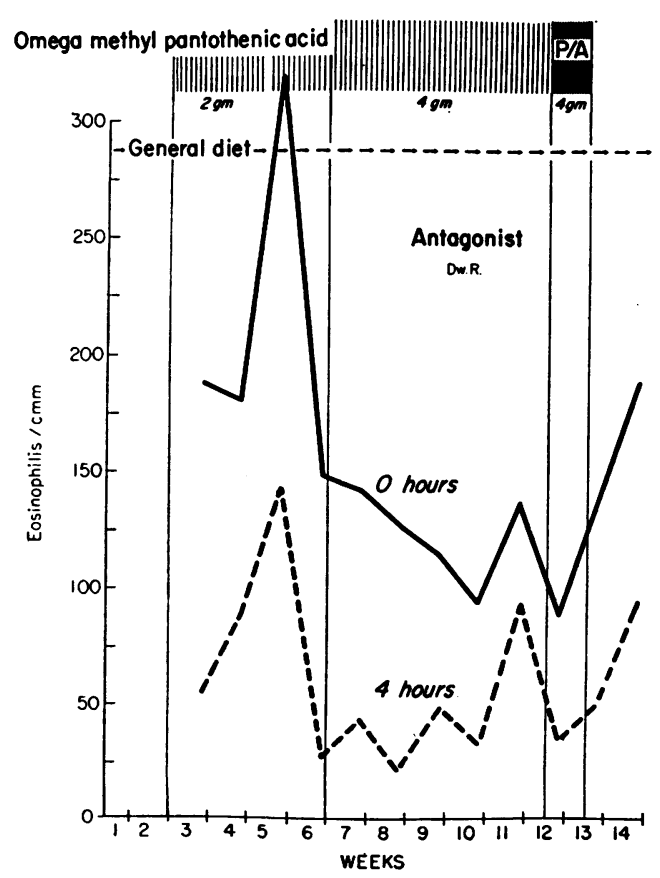

Fig. 5. Eosinopenic Response to Adrenocorticotropic HoRMONE (ACTH)

In Subject No. 4, the eosinopenic response was impaired during the eleventh week, but returned to normal when pantothenic acid was given. Note the relative eosinopenia which occurred after the sixth week. 


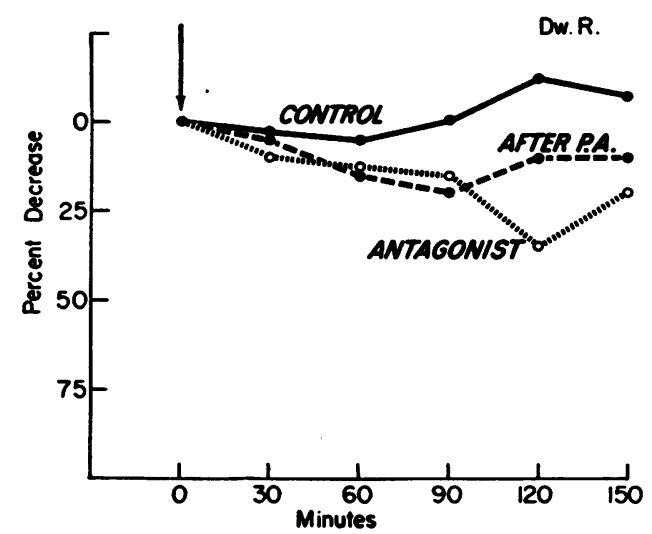

Fig. 6. Hypoglycemia After Insulin (0.1 Unit per Kg. Body Weight Subcutaneously)

Subject No. 4, who received the largest dose of antagonist, developed increased sensitivity to insulin. This was corrected partially by administration of pantothenic acid.

decrease in his total eosinophil count with gradual development of impaired responsiveness to ACTH (Figure 5). After the administration of pantothenic acid these abnormalities disappeared. $\mathrm{He}$ alone developed an exaggerated response to insulin (Figure 6), which returned toward normal when pantothenic acid was given. All four men remained in positive nitrogen balance throughout the study. Electrophoretic determinations of serum proteins disclosed no abnormalities.

\section{DISCUSSION}

Our demonstration that an abnormal state appeared in healthy young men fed a diet deficient in pantothenic acid together with the antagonist, omega-methyl pantothenic acid, left several questions unanswered $(1,2)$. In our first studies we had found that some subjects were hypokalemic and had the expected electrocardiographic changes $(3,4)$. Subsequently we learned that this resulted from the combined effects of the relatively small amount of potassium and large amount of bicarbonate in the formula (5). Hypokalemia was avoided by substituting sodium chloride for sodium bicarbonate and by increasing the potassium in the diet. Similarly, changes in carbohydrate tolerance apparently had resulted from the composition of the original formula which allowed rapid absorption of glucose resulting in a fast rise and fall of blood sugar. This was corrected by increasing the viscosity of the formula, starch replacing a portion of the glucose. Another apparent artifact was observed in the urinary excretion of 17 -ketosteroids which had decreased when large amounts of para-aminobenzoic acid inhibited their excretion in the urine. Previously we had found impairment of gastric secretion in patients who were deficient in pantothenic acid but this was not found in later studies (3-6).

After many trials and errors we were able to induce regularly a clearly defined illness in healthy young men by giving a diet deficient in pantothenic acid for a sufficiently long time. The symptoms and biochemical abnormalities in many but not all respects were similar to those which occurred in deficient animals. Furthermore the administration of large amounts of the active vitamin corrected these abnormalities in most instances.

In the present study many of the same symptoms and biochemical abnormalities occurred in healthy young men fed a balanced nutritious diet and given omega-methyl pantothenic acid. The abnormalities included impairment of the eosinopenic response to $\mathrm{ACTH}$ and hypersensitivity to insulin. The slight elevation of the erythrocyte sedimentation rate was considered of doubtful significance. The evaluation of symptoms, although depending upon subjective interpretations, was sufficiently consistent to warrant the conclusion that we were observing the same neurological and gastrointestinal disorders observed in previous tests. Although the fourth subject complained of no symptoms, his stoical character may have prevented him from making any complaint. $\mathrm{He}$ did develop a distinct tremor of his hands and biochemical abnormalities consistent with our previous findings in pantothenic acid deficiency. Administration of the active vitamin was followed by recovery from all signs and symptoms.

The differences between signs of vitamin deficiency in animals and in man are well known to all who have endeavored to compare them. Different species of animals vary in vitamins required and in reactions to deprivation. The inherent dissimilarities of animals and man, and the ethical and moral obligations which oblige a physicianinvestigator to be concerned for the welfare of his patients, add to the complexity of clinical trials. When only a few subjects can be studied, individual differences among subjects add a further complexity. Consequently a partial or in- 
complete state of deficiency may be induced in some subjects, while others remain well, or nearly so. To attempt to induce severe symptoms for protracted periods of time in all subjects might result in injurious or even fatal disease. These considerations made us proceed slowly, accept early and partial illness as evidence of deficiency, and restore adequate nutrition promptly after illness was established. The results were definite. The mechanisms remain obscure.

\section{SUMMARY}

Four healthy young men were fed a balanced 3,200 calorie diet containing $120 \mathrm{Gm}$. of protein. One man served as a control while the other three received different amounts of the antagonist, omega-methyl pantothenic acid. The control subject and the man who got the smallest amount of anti-vitamin remained in fairly good health. The man who received an intermediate dose developed gastrointestinal and neurologic symptoms characteristic of pantothenic acid deficiency. The fourth man, who received the largest dose, remained asymptomatic except for the development of tremor.

Biochemical abnormalities were found in the eosinopenic response to adrenocorticotropic hormone $(\mathrm{ACTH})$ of the two men who received the most antagonist, and in the sensitivity to insulin of the man who received 2 to $4 \mathrm{Gm}$. daily. We conclude that daily administration of 2 to $4 \mathrm{Gm}$. of omega-methyl pantothenic acid induced clinical and metabolic changes consistent with pantothenic acid deficiency even though a normal diet was fed. This illness was corrected by administration of the active vitamin after we stopped giving the antagonist.

\section{ACKNOWLEDGMENTS}

The authors wish to thank the volunteers and Warden Ray Purcell of the State Reformatory at Anamosa, Iowa. We also appreciate the painstaking assistance of the nurses, dietitians and technicians who so ably assisted us.

\section{REFERENCES}

1. Bean, W. B., and Hodges, R. E. Pantothenic acid deficiency induced in human subjects. Proc. Soc. exp. Biol. (N. Y.) 1954, 86, 693.

2. Bean, W. B., Hodges, R. E., and Daum, K. Pantothenic acid deficiency induced in human subjects. J. clin. Invest. 1955, 34, 1073.

3. Lubin, R., Daum, K. A., and Bean, W. B. Studies of pantothenic acid metabolism. Amer. J. clin. Nutr. 1956, 4, 420.

4. Bean, W. B., Lubin, R., and Daum, K. Studies of pantothenic acid metabolism. Trans. Amer. clin. Climat. Ass. 1955, 67, 73.

5. Hodges, R. E., Ohlson, M. A., and Bean, W. B. Pantothenic acid deficiency in man. J. clin. Invest. 1958, 37, 1642.

6. Thornton, G. H. M., Bean, W. B., and Hodges, R. E. The effect of pantothenic acid deficiency on gastric secretion and motility. J. clin. Invest. 1955, 34, 1085 . 